\title{
A Study To Determine Relation Between Chronic Tobacco Ingestion And Gastro-Intestinal Lesions In Western Uttar Pradesh : An Endoscopic Study.
}

\author{
Bhatnagar $\mathrm{M}^{*}$, Mishra DK**, Jha $\mathrm{S}^{* * *}$ \\ Pg Dept. Of Medicine, Subharti Medical College, Meerut \\ *Professor, Dept. Of Medicine, **Resident, Dept. Of Medicine, \\ *** Assistant Prof., Dept. Of Medicine
}

\section{Introduction}

\section{Introduction}

- Tobacco ingestion is a habit widely prevalent in India. Tobacco is ingested in many forms such as cigarette,bidi,chewingtobacco,hookah,khaini,Mawa and Dry Snuff etc. ${ }^{1}$

- The effect of chronic ingestion of tobacco on the gastrointestinal mucosa varies from ulcerations/erosions to the occurrence of rapidly progressive malignancies.

- The impact of consumption of different forms of tobacco on the GI mucosa, however, is still largely unknown

- This study was undertaken to find out the effect of different forms of tobacco consumption on the GI mucosa as compared with age and sex matched controls.

\section{Aims/Objectives :}

- To determine relation between chronic use of tobacco products and gastrointestinal lesions

- To study the effect of different types of products consumed on the GI mucosa.

Materials/Methods : 100 patients presenting to the Outpatient Dept. Of Medicine with symptoms of dyspepsia warranting Upper G.I Endoscopy were divided into two groups of 50 each(50 smokers/tobacco chewers and 50 non-smokers). All 100 patients underwent UGI Endoscopy and biopsy of visible lesion, if required.

Inclusion Criteria : Pts. were included if they matched the criteria of chronic smoker/ chronic tobacco chewer as follows: ${ }^{2}$

- Pack years > 1.5 i.e Smoking of more than 5 cigarettes/ day for at least the last 3 years.

- Chewing tobacco/ tobacco products such as - Khaini, Guthka, PaanMasala atleast 3 times/ day for the last 3 years

Exclusion criteria:

1.) Previously treated patients of any GI illness/ gastrointestinal mucosal pathology.

2.) Patients on co-existing usage of long term( $>6$ weeks) Steroids/ NSAIDS for any medical condition.

3.) Patients consuming Proton Pump Inhibitors $1 / \mathrm{H} 2$ blockers week prior to study.

4.) Patients suffering from gastritis due to other identifiable causes such as : Recent or chronic alcohol intake, chronic kidney disease etc.

Results : Of the 100 patients, 19 had normal endoscopy results, whereas 81 had significant findings. Amongst these $81,51(62.96 \%)$ were smokers/tobacco chewers. The other 30(37.04\%) did not consume any tobacco. In the 81 with significant findings, the majority of the lesions were present in the Gastric Antrum( $(41 \%)$ whereas the area least affected was middle $1 / 3^{\text {rd }}$ of oesophagus(1.1\%). 


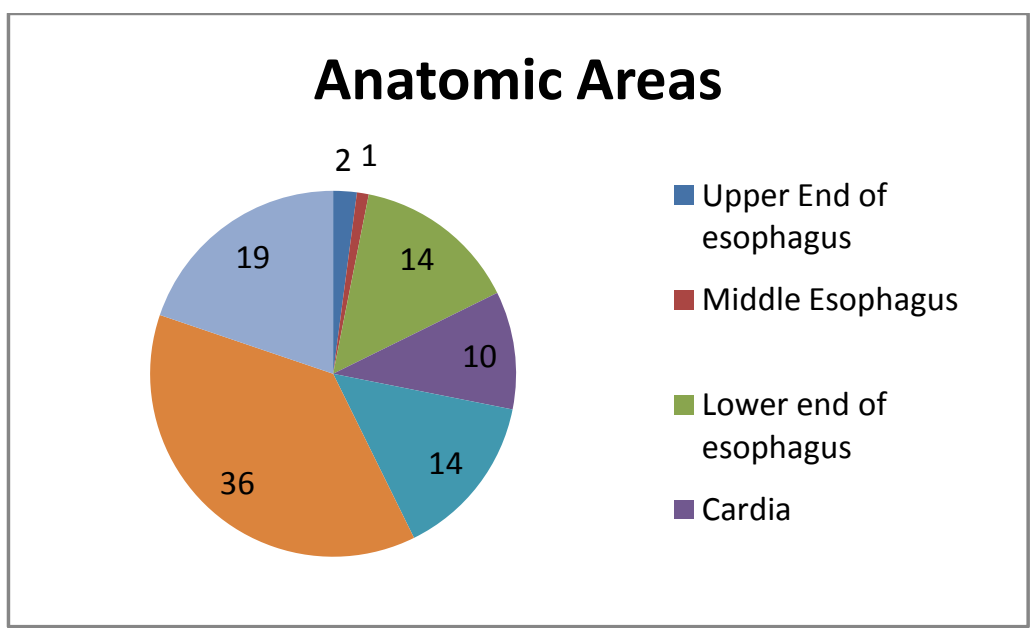

14 subjects had minor erosions and signs of non-specific inflammation, 47 had visible ulcerations without any bleeding, 8 subjects had visibly active bleeding/ signs of chronic inflammation like strictures and fibrosis and 12 subjects had a visible growth arising out of the gastric and esophageal mucosa, which were biopsied.

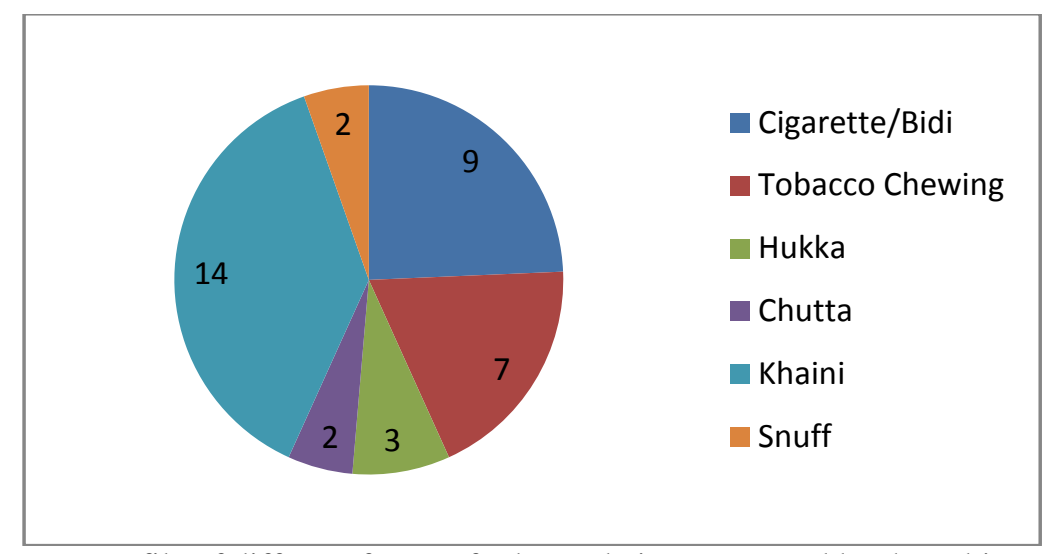

Profile of different forms of tobacco being consumed by the subjects $(\%)$

The profile of patients classified in each grade as evident on endoscopy was as follows :

\begin{tabular}{|l|l|l|l|l|l|l|}
\hline Grade & Total & Smokers & $\%$ & Non - Smokers & $\%$ & Difference \\
\hline A & 19 & 2 & $10.5 \%$ & 17 & $89.5 \%$ & $79 \%$ \\
\hline B & 14 & 5 & $35.7 \%$ & 9 & $64.3 \%$ & $28.6 \%$ \\
\hline C & 47 & 34 & $72 \%$ & 11 & $28 \%$ & $44 \%$ \\
\hline D & 8 & 5 & $62.5 \%$ & 3 & $37.5 \%$ & $25 \%$ \\
\hline E & 12 & 11 & $91.6 \%$ & 1 & $8.3 \%$ & $83.3 \%$ \\
\hline
\end{tabular}

Chi Square value $=31.81$.

$P$ value $=<0.001$ (significant)

In the tobacco intake group, the maximum endoscopic findings were present in the patients who were consuming more than 2 tobacco products at a time $(42.7 \%)$ but among isolated products, Hookah consumption seemed to cause the most damage to the GI mucosa(19.8\%). 


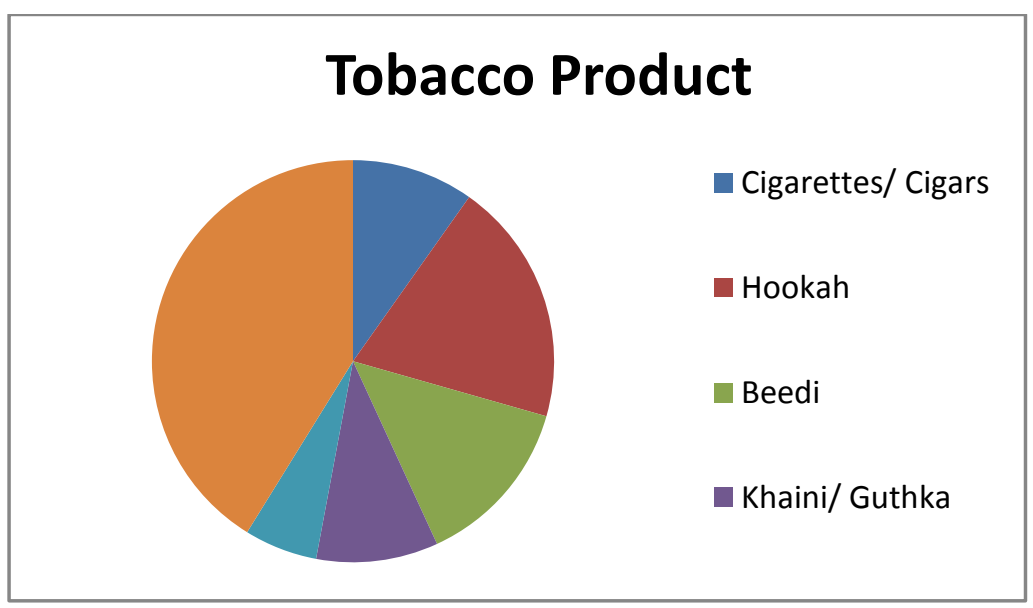

The total number of patients with endoscopically visible growth were 12.11 of these consumed tobacco in some form. 6 of these were microscopically proven to be malignant, 4 of which were squamous cell and 2 were adenocarcinoma.

\section{Discussion}

This work titled :'A study to determine relation between chronic use of tobacco products and gastrointestinal lesions : An Endoscopic Study.' was carried out in the Dept. Of Medicine , ChhatrapatiShivajiSubharti Hospital, Subharti Medical College, Meerut from December 2014 to June 2016. Smoking and ingestion of tobacco products are well known independent risk factors for gastrointestinal disorders.Epidemiological and experimental studies have shown that tobacco and its products lead to many harmful effects on the gastrointestinal mucosa, most common amongst which are gastro duodenal erosions, ulcers and gastric cancer. The mortality of peptic ulcer disease is also higher in smokers than non-smokers. Changes in the gastric histology mainly include inflammation of mucosa, polymorphonuclear infiltration and increased gastric mucosal cell apoptosis. Healing of ulcers and lesions is also significantly delayed in chronic smokers. The use of more than one tobacco product increases the risk for these, specially in men., 3,5

The present study was undertaken to find out the presence of gastrointestinal morbidity in patients who are chronic smokers/tobacco chewers and compare them with age and sex matched controls i.e those who do not consume tobacco in any form by performing upper gastrointestinal endoscopy.

50 smokers and 50 non-smokers who presented to the hospital with symptoms warranting an upper gastrointestinal endoscopy were age and sex matched and selected for the study on the basis of strict inclusion and exclusion criteria. Their biochemical and clinical parameters were evaluated and compared. After an informed consent, all of these patients underwent an upper gastrointestinal endoscopy.

The study comprised of 50 smokers/tobacco chewers and 50 non-smokers. The total number of males was 58 while the females enlisted were 42 . The age of the patients ranged from a minimum of 18 years to a maximum of 86 years with the mean age being 52.7 years. Each group(smokers and non-smokers) had 31 males and 19 females each. The age and sex difference was found to be statistically insignificant.

All patients then underwent upper gastrointestinal endoscopy. 19 patients out of 100 had normal endoscopic findings whereas 81 patients had significant endoscopic findings. Grade I changes were visible in a total of $14(16.6 \%)$ patients whereas grade II changes were seen in a majority $47(55 \%)$ of cases. Of the 81 significant endoscopic findings, 51(62.96\%) were found in smokers whereas $30(37.04 \%)$ were found in nonsmokers. Joel Richter in his study had demonstrated that tobacco smokers and tobacco chewers were much more susceptible to developing GERD, oesophageal stenosis and inflammation of the gastrointestinal tract. ${ }^{4}$

A large number of the patients,36(41\%) had findings more prominent in the Antrum of stomach whereas findings were least present in the middle $1 / 3^{\text {rd }}$ of the oesophagus. Mag,Tarm et. Al had in 2011 shown in their study that among 989 smokers and tobacco chewers who underwent upper GI endoscopy, 95\% patients had endoscopically visible lesions, $45 \%$ of which were present in the antrum of stomach alone.

Namoit Z in 2007 had demonstrated that cigarette smoking causes neutrophilic infiltration and caused, in specific, antral inflammation and atrophy. ${ }^{6}$ So, our findings are in accordance with previous literature. $22(25.2 \%)$ of the patients had endoscopically visible changes in the first and second part of the duodenum , which is also closely associated with the chronic ingestion of tobacco and causes inflammation of the entire thickness of the duodenal wall. Hull, DH et Al. had shown in 30 chronic smokers that long term nicotine intake can cause a significant increase in the round cell and eosinophil count in the duodenum thereby causing duodenal inflammation. ${ }^{7}$ 
12(14.2\%) patients who underwent endoscopy also had growths/masses which were endoscopically visible and were subsequently biopsied. Only 1 of these 12 was a non-smoker whereas the other 11 consumed tobacco in some form or other. Out of these 12, 6 biopsy samples proved to be malignant, which shows a very high incidence rate of gastrointestinal malignancy in persons who consume tobacco.

Ahmed,W. et $\mathrm{Al}$ had found that in 383 patients who were chronically ingesting tobacco, $78 \%$ had a endoscopically visible lesion. While the most common lesion was a duodenal ulcer, a large number of patients also did have malignant growths and tumours. ${ }^{8}$ Sonja P. Dawsey et Al also found in their study of 109 young patients that tobacco smoking was the single largest independent risk factor causing malignancies of the gastrointestinal tract.Among the 6 patients with biopsy proven malignancy, 4(66\%) had squamous cell carcinoma whereas $2(34 \%)$ of the patients had adenocarcinoma of the gastrointestinal tract. Numerous studies and research articles have now proven that while adenocarcinoma is on the rise in western countries, in developing countries such as India, it is squamous cell carcinoma which has a higher incidence and prevalence thereby causing a higher mortality. ${ }^{9}$

No significant difference in gender was noted for the occurrence of gastrointestinal malignancy, though. Dar NA, Bhatt GA et Al. demonstrated in 702 patients who had endoscopic and biopsy proven malignancy that squamous cell carcinoma was much more prevalent amongst cigarette and hookah smokers. ${ }^{10}$

The study has shown that although dyspepsia, dysphagia and other complaints maybe present in smokers and non-smokers both, the severity of gastrointestinal morbidity in the form of ulcers,erosions, GI bleeding etc. is significantly higher in persons who consume tobacco on a chronic basis. Also, the incidence of malignancy, squamous cell and adenocarcinoma both appears to be significantly higher in persons who intake tobacco in any form.

Smedley et Al. had in their retrospective study of 275 smokers and 275 non-smokers discovered that cigarette smokers were much more likely to suffer from chronic peptic ulcers, stenosis of lumen and fibrosis of mucosa etc. Of the 128 patients with duodenal ulcers in his study, 85.9\% were smokers. A similar pattern was found in our study as well, where $71.9 \%$ of the subjects suffering from chronic peptic ulcers were smokers. ${ }^{11}$

In their study of 90 patients with biopsy proven squamous cell carcinoma, Chitra S et. Al found that bidi and cigarette smoking and paan/tobacco chewing are all independent risk factors for the development of oesophageal carcinoma. They also found that the risk for development of cancer was 2.8 and 2.5 times higher in the smokers and tobacco chewers respectively. These findings were corroborated in our study where $91.66 \%$ of patients who were diagnosed with biopsy proven malignancy were either chronic smokers or tobacco chewers. In addition, $67 \%$ of these patients had squamous cell carcinoma of the gastrointestinal tract. ${ }^{12}$

\section{Conclusions}

1.) The present study has shown a very high association of chronic tobacco intake with the development of endoscopically visible gastrointestinal disease. The rate of development of gastrointestinal malignancy was also found to be significantly higher in patients who were chronic tobacco smokers/chewers.

2.) The study found chronic tobacco ingestion in any form(cigarette,hookah,chewable tobacco) etc. to be equal and independent risk factors for the development of gastrointestinal inflammation, erosions, ulcers and malignancies.

\section{References}

[1]. Kulkarni R, Ujwal K et Al. - A Clinico Pathological Study of Upper Gastro Intestinal Endoscopy in Patients with Dyspepsia. Int J Sci Res.2016;4:2319-7064

[2]. American Lung Association Criteria for chronic smoking. http://www.lung.org/lung-health-and-diseases/

[3]. World WHO Databank 2012http://data.worldbank.org/indicator/SH.PRV.SMOK.MA]

[4]. Richter J. Advances in GERD and Current Developments in the Management of Acid-Related GI Disorders. GastroenterolHepatol (N Y).2010 Feb; 6(2): 76-79.

[5]. Aro, P., Ronkainen, J., Storskrubb, T. et al. A study to determine relation between tobacco and gastrointestinal morbidity on endoscopy - Eur J Epidemiol (2010) 25: 741.

[6]. Namiot Z, Namiot DB, Kemona A, Gołebiewska M, Bucki R. The effect of cigarette smoking and alcohol consumption on efficacy of Helicobacter pylori eradication. Pol Arch Med Wewn. 2000;104(3):569-574.

[7]. Hull DH, Beale PJ. Relation between cigarette smoking and duodenal ulcer on endoscopy. Gut.1985;26(12):1333-1337.

[8]. Ahmed, W., Qureshi, H. Association of upper gastrointestinal lesions with addictions. JPMA.1993, 43(9):176-177.

[9]. Dawsey SP, Tonui S, Parker RK, Fitzwater JW, Dawsey SM, White RE, et al. (2010) Esophageal Cancer in Young People: A Case Series of 109 Cases and Review of the Literature. PLoS ONE 5(11): e14080. doi:10.1371/journal.pone.0014080

[10]. Dar NA, Islami F, Bhat GA, et al. Poor oral hygiene and risk of esophageal squamous cell carcinoma in Kashmir. Br J Cancer. 2013;109(5):1367-1372

[11]. Smedley F, Hickish T, Taube M, Yale C, Leach R, Wastell C. 1988. Perforated duodenal ulcer and cigarette smoking. J R Soc Med.1988;81(2):92-94.

[12]. Chitra S, Ashok L et al. Risk factors for oesophageal carcinoma in Coimbatore,South India : A hospital based case control study. Indian J Gastroenterol. 2004;23:19--21. 